\title{
Destruxin B Isolated from Entomopathogenic Fungus Metarhizium anisopliae Induces Apoptosis via a Bcl-2 Family-Dependent Mitochondrial Pathway in Human Nonsmall Cell Lung Cancer Cells
}

\author{
Chun-Chi Wu, ${ }^{1,2}$ Tzu-Hsiu Chen, ${ }^{3}$ Bing-Lan Liu, ${ }^{4}$ Li-Chen Wu, ${ }^{5}$ Yung-Ching Chen, \\ Yew-Min Tzeng, ${ }^{4}$ and Shih-Lan Hsu ${ }^{1,6}$ \\ ${ }^{1}$ Institute of Medicine, Chung Shan Medical University, Taichung, Taiwan \\ ${ }^{2}$ Department of Medical Research, Chung Shan Medical University Hospital, Taichung, Taiwan \\ ${ }^{3}$ Department of Health and Nutrition, Chia Nan University of Pharmacy \& Science, Tainan, Taiwan \\ ${ }^{4}$ Department of Applied Chemistry, Chaoyang University of Technology, Taichung, Taiwan \\ ${ }^{5}$ Department of Applied Chemistry, National Chi Nan University, Puli, Nantou, Taiwan \\ ${ }^{6}$ Department of Education and Research, Taichung Veterans General Hospital, Taichung, Taiwan
}

Correspondence should be addressed to Yew-Min Tzeng; ymtzeng@cyut.edu.tw and Shih-Lan Hsu; h2326@vghtc.gov.tw

Received 20 March 2013; Accepted 20 August 2013

Academic Editor: Mei Tian

Copyright (C) 2013 Chun-Chi Wu et al. This is an open access article distributed under the Creative Commons Attribution License, which permits unrestricted use, distribution, and reproduction in any medium, provided the original work is properly cited.

\begin{abstract}
Destruxin B, isolated from entomopathogenic fungus Metarhizium anisopliae, is one of the cyclodepsipeptides with insecticidal and anticancer activities. In this study, destruxin B was extracted and purified by ion-exchange chromatography, silica gel chromatography, and semipreparative high-performance liquid chromatography. The potential anticancer effects and molecular mechanisms of destruxin B in human nonsmall cell lung cancer cell lines were characterized. Our results showed that destruxin B induced apoptotic cell death in A549 cells. This event was accompanied by the activation of caspase-2, -3 , and -9 . Moreover, destruxin B increased the expression level of proapoptotic molecule, PUMA, while decreased antiapoptotic molecule Mcl-1. Additionally, the translocation of Bax from cytosol to mitochondrial membrane was observed upon destruxin B treatment. Knockdown of Bax by shRNA effectively attenuated destruxin-B-triggered apoptosis in A549 cells. Interestingly, similar toxic effects and underlying mechanisms including caspase activation, upregulation of PUMA, and downregulation of Mcl-1 were also observed in a p53-null lung cancer H1299 cell line upon destruxin B treatment. Taken together, our findings suggest that destruxin-B-induced apoptosis in human nonsmall cell lung cancer cells is via a Bcl-2 family-dependent mitochondrial pathway.
\end{abstract}

\section{Introduction}

Lung cancer is the leading cause of death for both men and women in many countries, including Taiwan, which exhibited the highest rate of increase in lung cancer mortality in recent years $[1,2]$. The five-year survival rate of lung cancer patient is only $13-25 \%$ [3]. Although target therapy emerged for treatment of lung cancer patients in the past decade [4], there is still a large amount of patients who are uncured. It appears that the drugs with greater efficacy than existing treatments are urgently required for these patients.
Growing evidence demonstrates that apoptosis is involved in many biological events including physiological homeostasis and embryogenesis [5] and is also an important determinant of the response of cancers to chemo-, radiation, or target-therapy [6,7]; compounds that induce these events may provide potent anticancer effects for cancer treatment. The regulatory mechanisms of apoptosis are quite complicated networks. Two major types of apoptosis pathways are mentioned: one is the intrinsic pathway which is mediated by the regulators located in the mitochondrial [8] and the other is the extrinsic pathway which is regulated 
by the binding of the membrane receptor to the death ligand such as TNF (tumor necrosis factor)/TNFR and Fas/FasL [9]. The apoptotic process is mainly executed by a class of cysteine proteases known as caspases [10]. Activation of different caspases usually presents for various upstream apoptotic pathways. For example, the activation of caspase- 8 and -10 usually stands for the extrinsic/death receptor-related pathway; caspase- 2 and caspase- 9 are usually activated by mitochondrial pathway, while caspase- 12 is activated by endoplasmic reticulum pathway [10]. In addition, the activation of caspase-3, -6 , or -7 is usually considered as a common executor of apoptosis by directly cleaving cellular proteins when activated [11].

Several proteins are known to be involved in the regulation of apoptosis; among them $\mathrm{Bcl}-2$ family molecules contribute to the mitochondrial-mediated apoptotic pathway. $\mathrm{Bcl}-2$ is originally found in a translocation chromosomal fragment in B-cell lymphoma and is proved as a protooncogene [12]. The members of $\mathrm{Bcl}-2$ family all contain a $\mathrm{BH}$ (Bcl-2 homology) domain structure and are divided into two major groups: one composed of antiapoptotic molecules, including Bcl-2, Bcl-xL, Bcl-w, Mcl-1, Boo/Diva, and A1/Bfl1 [13] and the other comprises proapoptotic molecules, such as Bax, Bak, Bad, Bim, Bik, PUMA, and NOXA [14]. Under normal conditions, the $\mathrm{Bcl}-2$ or $\mathrm{Bcl}-\mathrm{xL}$ complexes with Bax or Bak to form homo- or hetero dimer, and such interaction will neutralize proapoptotic molecules to prevent cell death. Under apoptotic stimulation, however, it will change the ratios between these two groups, leading to the formation of Bax/Bax or Bax/Bak complexes followed by perforation through the membrane of mitochondria. The opened pores eventually cause the release of cytochrome c from mitochondria to the cytosol and form apoptosis complex with Apaf-1 (apoptotic protease activation factor1), dATP (deoxyadenosine triphosphate), and procaspase9 to induce the caspase- 9 and caspase- 3 activation and irreversible apoptosis [15].

It is well known that certain compounds isolated from bacteria or fungus carry characteristic biological effects, which have been used as traditional medicines [16, 17], and several of these compounds have shown an antitumoral activity [18-20]. One group of such biological active compounds is depsipeptides, which are the major secondary metabolites of fungi. The depsipeptides are biooligomers formed by esterification between hydroxyl groups and amino acids. Destruxin B is a cyclic depsipeptide isolated from entomopathogenic fungus in 1961. Until recently, there were 39 destruxins reported [21]. It has been reported that cyclic depsipeptides exhibited strong antitumor activity in both in vitro and in vivo experiments and evenly entered the clinical trial [22]. Previous reports mentioned that destruxin B has strong anti-insect activity [23], inhibits hepatitis B surface antigen expression, and induces cell death in hepatocellular carcinoma cells [24] and leukemic cells [25]. The effects of destruxin B on lung cancer cells and its molecular mechanisms, however, have not been examined yet. In this study, the antiproliferative, cytotoxic effects and underlying mechanisms of destruxin B (isolated and purified from fermentation broth of Metarhizium anisopliae) on lung cancer cell lines were explored. Our observations indicate that destruxin B induces a Bcl-2 family-dependent mitochondrial apoptotic cell death in human nonsmall cell lung cancer A549 and H1299 cell lines.

\section{Material and Methods}

2.1. Reagents. The destruxin B (MW 593) was dissolved in ethanol to a stock solution at concentration of $20 \mathrm{mM}$ for the following experiments. Anti-Bax, Bcl-2, Bcl-xL, Bak, PUMA, Mcl-1, and $\beta$-actin were purchased from Santa Cruz Biotechnology (Santa Cruz, CA, USA) or Calbiochem Chemical Company (CN Biosciences, Notts, UK). Anti-mouse and anti-rabbit IgG peroxidase-conjugated secondary antibodies were purchased from Amersham (Buckinghamshire, UK). Caspase activity assay kits, including the fluorogenic substrates of caspase-2 (VDVAD-AFC), caspase-3 (DEVD-AFC), and caspase-9 (LEHD-AFC), were purchased from R\&D systems (Minneapolis, MN, USA). The caspase-2 inhibitor (z-VDVAD-FMK) was purchased from Santa Cruz Biotechnology (Santa Cruz, CA, USA). The caspase-3 inhibitor (ZDEVD-FMK) and caspase-9 inhibitor (Z-LEHD-FMK) were purchased from Kamiya (Seattle, WA, USA).

2.2. Isolation and Purification of Destruxin B. The destruxin $B$ used in this study was extracted and purified from the submerged cultivation of fungus Metarhizium anisopliae F061 var. Anisopliae according to the processes described elsewhere [26, 27]. Briefly, Metarhizium anisopliae was cultivated in $3 \%$ maltose containing $0.5 \%$ bactopeptone liquid medium, at $28^{\circ} \mathrm{C}$. After 14 days, the culture medium was collected and extracted three times with equal volume of methylene dichloride $\left(\mathrm{CH}_{2} \mathrm{Cl}_{2}\right)$. The organic layer was collected and concentrated and further separated by two-step ion-exchange chromatography. Combination of cationic (Amberlite TRA$400 \mathrm{CP}$ column) and anionic (Amberlite IR 120 Plus) exchange provided the step to removing charged impurities existing in the mixture, and then the eluent was collected and subjected to flash silica chromatography (Merck K-60 silica gel column) as a prepurification step. The column was eluted with a methylene dichloride-methanol mixture. Further purification was done by loading the sample into a semipreparative RP-C18 high-performance liquid chromatographic (HPLC) column. The which elution delivered a single peak was collected and concentrated. This peak represented destruxin $B$ (retention time $=17.6 \mathrm{~min}$ ). The purity of destruxin B in the sample was proved $>95 \%$ in our early report [28].

2.3. Cell Culture and Viability Assay. The human lung cancer A549 and H1299 cells were obtained from CCRC (Culture Collection and Research Center, Hsinchu, Taiwan). The A549-shBax cells were established by transfection of pSupershBax followed by antibiotics selection [29]. A549, A549shBax, and H1299 cells were cultured in RPMI (GIBCO/BRL, MD, USA). All cultured media were supplemented with $10 \%$ fetal bovine serum, $2 \mathrm{mM}$ glutamine, and antibiotics (100 U/mL penicillin and $100 \mu \mathrm{g} / \mathrm{mL}$ streptomycin) at $37^{\circ} \mathrm{C}$ 
in a humidified atmosphere of $5 \% \mathrm{CO}_{2}$. The medium was changed every $48 \mathrm{~h}$. For cell viability assay, cells were seeded in 12 -well plates at a density of $1 \times 10^{5}$ cells per well. After $24 \mathrm{~h}$, cells were treated with various concentrations of destruxin $B$ for the indicated time points. After treatment, the number of viable cells was determined by trypan-blue dye exclusion method.

2.4. Cell Cycle Analysis. Cells were seeded as a number of $2 \times 10^{5}$ cells/well onto 6 -well plates. After $24 \mathrm{~h}$ incubation, different concentrations of destruxin $\mathrm{B}(0,10,20$, and $30 \mu \mathrm{M})$ were added for another $48 \mathrm{~h}$. The cells were then collected and washed with cold PBS twice followed by centrifugation at $1200 \mathrm{rpm}$ at $4^{\circ} \mathrm{C}$. Cell pellet was suspended with $1 \mathrm{~mL}$ cold ethanol (70\%) and then was incubated with buffer containing propidium iodide $(40 \mu \mathrm{g} / \mathrm{mL})$ and RNase A $(100 \mu \mathrm{g} / \mathrm{mL})$ for 30 min under dark condition. The prepared cell samples were subjected to flow cytometry (FACScan) and analyzed by ModFIT LT software.

2.5. Caspase Activity Assay. Cell lysates obtained from destruxin-B-treated or -untreated cells were tested for caspase-2, -3, -9 activities by addition of a caspase-specific peptide substrate conjugated with the fluorescent reporter molecule 7-amino-4-trifluoromethyl coumarin (R\&D Systems, Minneapolis, USA). The cleavage of the peptide by the caspase releases the fluorochrome that when excited by light at $400 \mathrm{~nm}$ emits fluorescence at $505 \mathrm{~nm}$. The level of caspase enzymatic activity in the cell lysate is directly proportional to the fluorescence signal detected using a fluorescent microplate reader (Fluoroskan Ascent; Labsystems, Finland).

2.6. Protein Preparation and Immunoblotting. Cells were cultured without or with destruxin B at various time points. After treatment, both adherent and floating cells were harvested, washed twice with ice-cold PBS, and decomposed in ice-cold modified RIPA buffer $(50 \mathrm{mM}$ Tris$\mathrm{HCl}$ ( $\mathrm{pH}$ 7.4), $150 \mathrm{mM} \mathrm{NaCl}, 1 \%$ Triton X-100, $0.25 \%$ Na-deoxycholate, $5 \mathrm{mM}$ ethylenediamine-tetra-acetic acid $(\mathrm{pH} 8.0), 1 \mathrm{mM}$ ethylene glycol-bis(2-aminoethylether)$\mathrm{N}^{\prime}, \mathrm{N}^{\prime} \mathrm{N}, \mathrm{N}^{\prime}$-tetra-acetic acid (pH 8.0), $1 \mathrm{mM}$ dithiothreitol, $0.2 \mathrm{mM}$ phenylmethylsulfonylfluoride, $5 \mu \mathrm{g} / \mathrm{mL}$ leupeptin, $5 \mu \mathrm{g} / \mathrm{mL}$ aprotinin, and $1 \mathrm{mM}$ sodium orthovanadate). After $30 \mathrm{~min}$ incubation on ice, cells were centrifuged at 100,000 g for $30 \mathrm{~min}$ at $4^{\circ} \mathrm{C}$, and supernatants were collected. Protein concentration was determined using the Bradford method. For western blot analysis, equal amounts of total proteins were loaded onto SDS-polyacrylamide gels followed by electrophoretically transferring onto a PVDF membrane (Millipore, MA, USA). Membranes were incubated with specific primary antibodies overnight. After exposure to horseradish peroxidase-conjugated secondary antibody for $1 \mathrm{~h}$, proteins were visualized using an enhanced chemiluminescence detection kit (ECL Kits; Amersham Life Science, NJ, USA).

2.7. Indirect Immunofluorescence. A549 cells were trypsinized and seeded onto coverslips. After reaching $75 \%$ confluence, cells were treated with destruxin B for $24 \mathrm{~h}$. Cells were washed with PBS twice and stained with MitoTracker for 15-30 min. After treatment, cells were washed with PBS and fixed with ethanol at $-20^{\circ} \mathrm{C}$ for $20 \mathrm{~min}$, incubated with Bax antibody at room temperature for $4 \mathrm{~h}$, and then washed three times with PBS and incubated with goat-anti-rabbitFITC (Jackson, PA, USA) and $1 \mathrm{mg} / \mathrm{mL}$ DAPI (Sigma, MO, USA) for another $1 \mathrm{~h}$. After washing with PBS containing $0.1 \%$ Tween 20 , the samples were mounted. The fluorescence images of the stained cells were analyzed using a Zeiss fluorescence microscope.

2.8. RNA Extraction and Quantitative RT-PCR. Total cellular RNA was extracted by RNA-Bee RNA isolation kit (TELTEST, Friendswood, TX) according to the manufacturer's instructions. One microgram of total RNA was reversetranscribed using Advantage RT for PCR Kit (Clontech, CA, USA) at $42^{\circ} \mathrm{C}$ for $1 \mathrm{hr}$. The PCR primers were listed as follows for Mcl-1 forward primer: AAAGAGGCTGGGATGGGTTT and reverse primer: CAAAAGCCAGCAGCACATTC; for PUMA forward primer: CCTGGAGGGTCCTGTACAATCT and reverse primer: GCACCTAATTGGGCTCCATCT; for $\beta$-actin forward primer: GCAAATGCTTCTAGGCGGACTAT and reverse primer: TGCGCAAGTTAGGTTTTGTCA. The mRNA levels were determined by real-time PCR with ABI PRISM 7900 Sequence Detector system according to the manufacturer's instructions. $\beta$-Actin was used as endogenous control. PCR reaction mixture contained the SYBR PCR master mix (Applied Biosystems), cDNA, and the primers. Relative gene expression level (the amount of target, normalized to endogenous control gene) was calculated using the comparative Ct method formula $E^{-\Delta \Delta \mathrm{Ct}}$.

2.9. Statistical Analysis. Figures were obtained from at least 3 independent experiments with similar patterns. All data are presented as mean \pm S.D. of 9 replicates from 3 separate experiments. Statistic differences were evaluated using Student's $t$-test, with ${ }^{*} P<0.05,{ }^{* *} P<0.01$, and ${ }^{* * *} P<0.001$ considered significant, or by the calculation and grouping using SAS program.

\section{Results}

3.1. Preparation of Destruxin B. Destruxin was isolated, purified, and identified from the fermentation broth of submerged cultivation of fungus Metarhizium anisopliae F061 var. Anisopliae by $\mathrm{CH}_{2} \mathrm{Cl}_{2}$ extraction, ion-exchange chromatography, silica gel chromatography, and HPLC quantification. Figure 1(a) presents a schematic diagram of extraction and isolation procedures. An HPLC fingerprint chromatogram was performed for the quantification and quality control of the isolated samples (Figure 1(b)), which showed only one major peak (retention time $=17.6 \mathrm{~min}$ ). The peak was identified as destruxin B. The purity of destruxin B in the sample was proved $>95 \%$ in our early report [28]. The chemical structure of destruxin B is presented in Figure 1(c). 


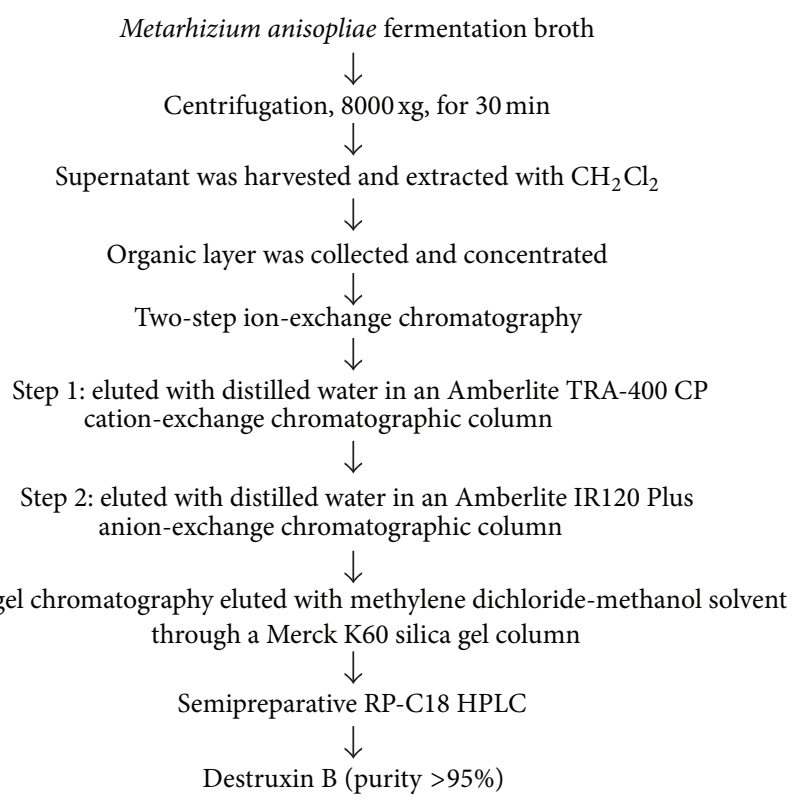

(a)

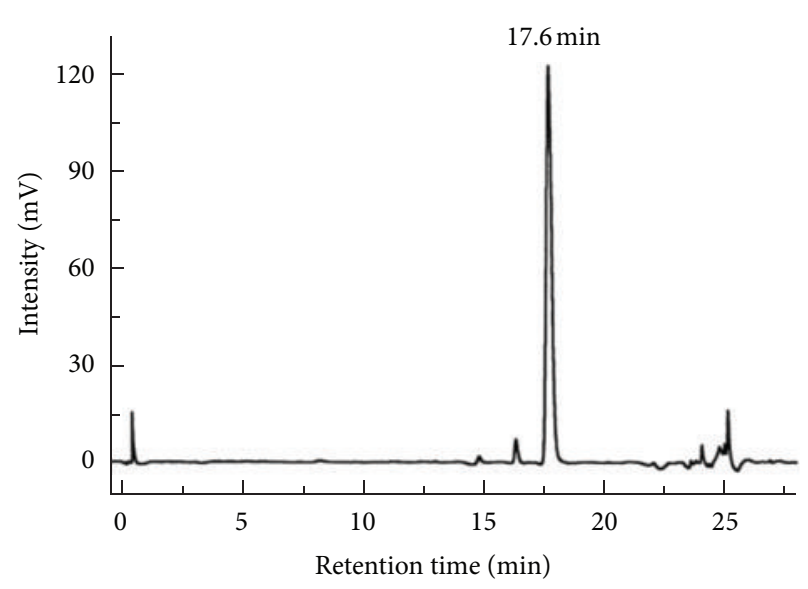

(b)<smiles>CC[C@H](C)[C@H](NC(=O)[C@H]1CCCN1C(=O)[C@H](CC(C)C)OC(=O)CCNC(=O)[C@H](C)N(C)C(=O)[C@@H](C(C)C)N(C)C(=O)[C@H](C)CC)C(=O)N1CCC[C@@H]1C</smiles>

(c)

FIGURE 1: Isolation and purification of destruxin B from Metarhizium anisopliae fermentation broth. (a) Flowchart of the extraction and purification procedure. (b) HPLC chromatographic spectrum of purified destruxin B. Metarhizium anisopliae fermentation broth was centrifuged and filtered. The filtrate was extracted with $\mathrm{CH}_{2} \mathrm{Cl}_{2}$, and then the destruxin $\mathrm{B}$ was extracted and purified by ion-exchange chromatography, silica gel chromatography, and HPLC. (c) The chemical structure of destruxin B.

3.2. Destruxin B Induces a Caspase-Dependent Apoptosis. To examine the growth inhibitory effect of destruxin $B$, different doses of destruxin $\mathrm{B}(0,1,5,10,20$, and $30 \mu \mathrm{M})$ were added into lung adenocarcinoma A549 cells. As shown in Figure 2(a), destruxin B induced a concentration-dependent antiproliferative effect on A549 cells, with $\mathrm{IC}_{50}$ value at $4.9 \mu \mathrm{M}$. Treatment with low concentrations of destruxin $\mathrm{B}$ resulted in growth inhibition but not cell death. However, destruxin-B-induced cytotoxicity occurred at concentration up to $10 \mu \mathrm{M}$ (Figure 2(a)). Data from cell flow cytometric analysis showed that destruxin B induced a sub-G1 population accumulation at 10 30 $\mu \mathrm{M}$ destruxin $\mathrm{B}$ administration (Figure 2(b)), indicating that destruxin B triggered the apoptotic cell death of A549 cells. To address whether destruxin-Binduced apoptosis was through a caspase activation pathway, fluorogenic peptide substrates were used to examine the specific caspase activity. As depicted in Figure 2(c), caspase$2,-3$, and -9 were significantly activated after $48 \mathrm{~h}$ destruxin incubation, while caspase- 8 and caspase- 12 were not activated upon destruxin B treatment (data not shown). To confirm the role of caspase activation in destruxin-B-mediated apoptosis, A549 cells were pretreated with caspase inhibitors prior to destruxin B. In Figure 2(d), the inhibitors of caspase-2, caspase-3, and caspase- 9 drastically attenuated destruxin-Binduced cell death, whereas the inhibitors of caspase- 8 and caspase-12 did not affect the destruxin-induced cell death (data not shown). These observations indicate that activation of mitochondria-dependent caspase cascade might play an important role in destruxin-B-induced apoptotic cell death in A549 cells. 


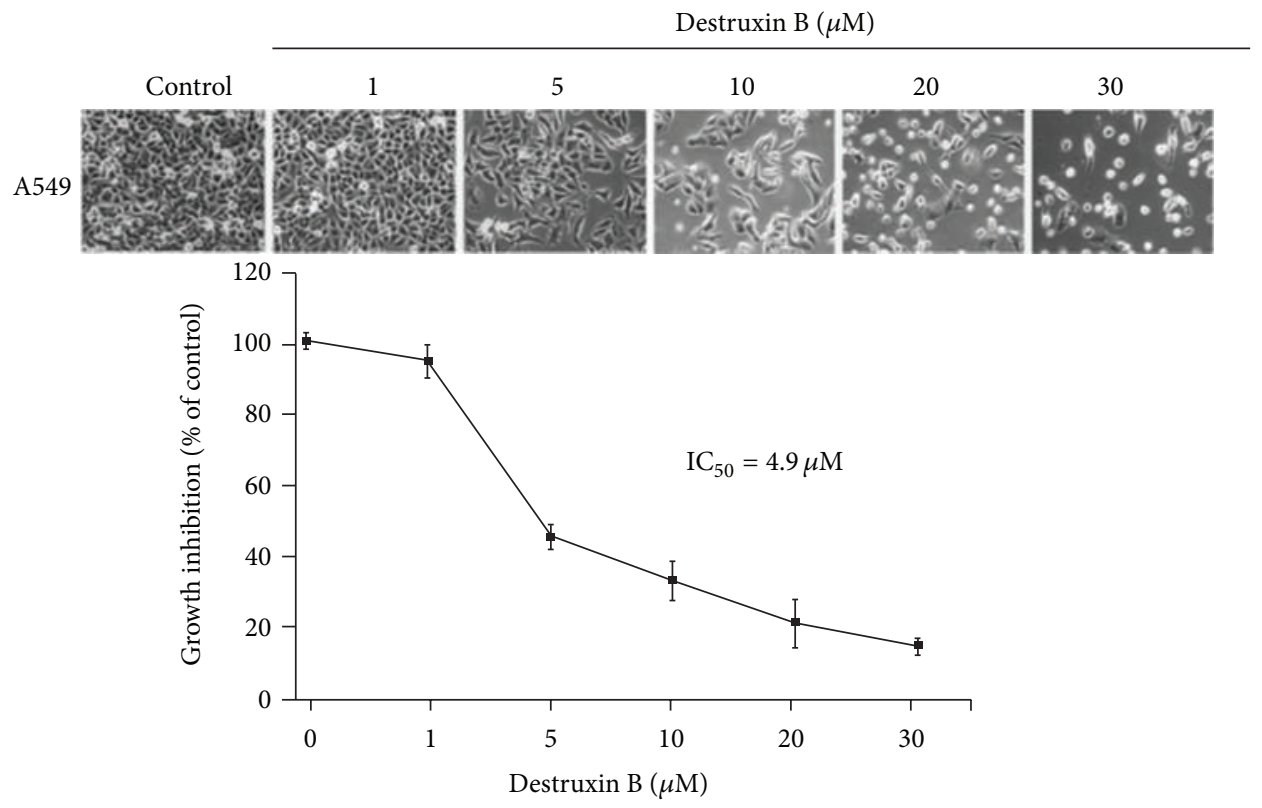

(a)
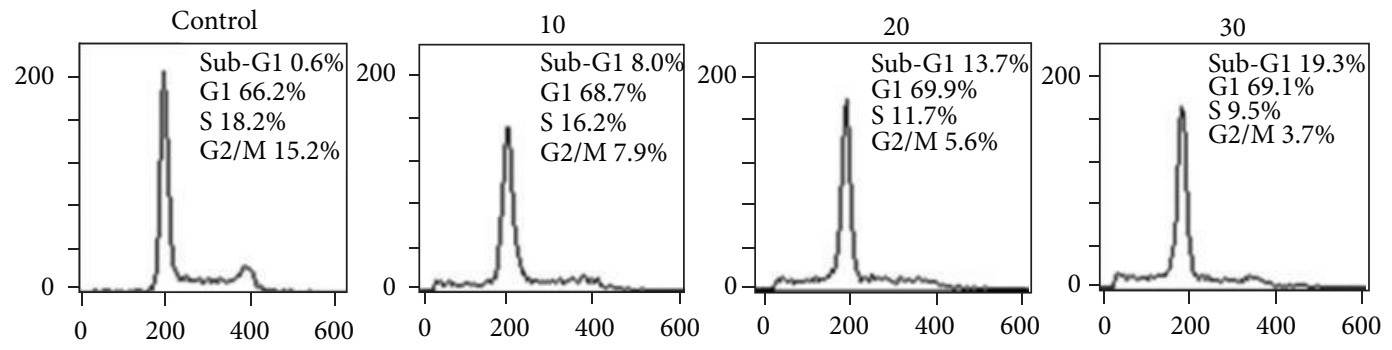

(b)

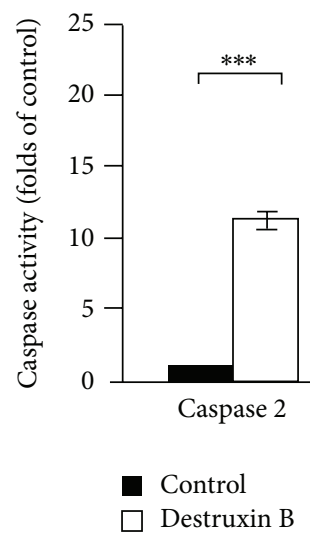

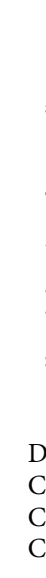

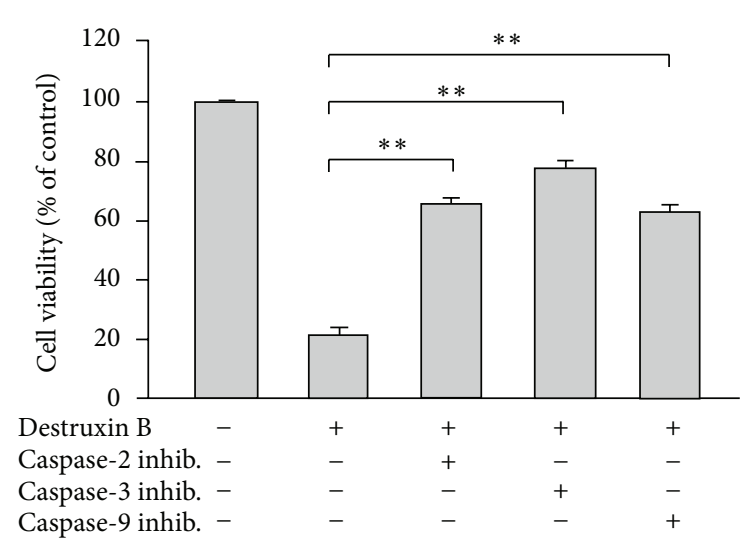

(c)

(d)

Figure 2: Destruxin B induces a caspase-dependent cell death. (a) Antiproliferative effect of destruxin B. Human nonsmall cell lung cancer A549 cells were administrated with different concentrations of destruxin B $(0,1,5,10,20$, and $30 \mu \mathrm{M})$ for $48 \mathrm{~h}$. The cellular morphology was observed under microscope and the cell numbers were then counted by trypan-blue dye exclusion method using hemocytometer. (b) SubG1 population is increased by destruxin administration. A549 cells were treated with different concentrations of destruxin B (0, 10, 20, and $30 \mu \mathrm{M}$ ) for $48 \mathrm{~h}$. Cells were then fixed with ethanol and subjected to flow cytometry assay. (c) Destruxin B induces caspase activation. A549 cells were treated with $20 \mu \mathrm{M}$ destruxin B for $48 \mathrm{~h}$. Cell lysates were harvested and the indicated caspase activity was measured as described in Section 2. (d) Inhibition of caspase activity blocks destruxin-B-triggered cell death. A549 cells were pretreated with indicated caspase inhibitors for $1 \mathrm{~h}$ and then treated with $20 \mu \mathrm{M}$ destruxin B for another $48 \mathrm{~h}$. The cell viability was determined by trypan-blue dye exclusion methods $\left({ }^{* *} P<0.01\right.$ versus control). 


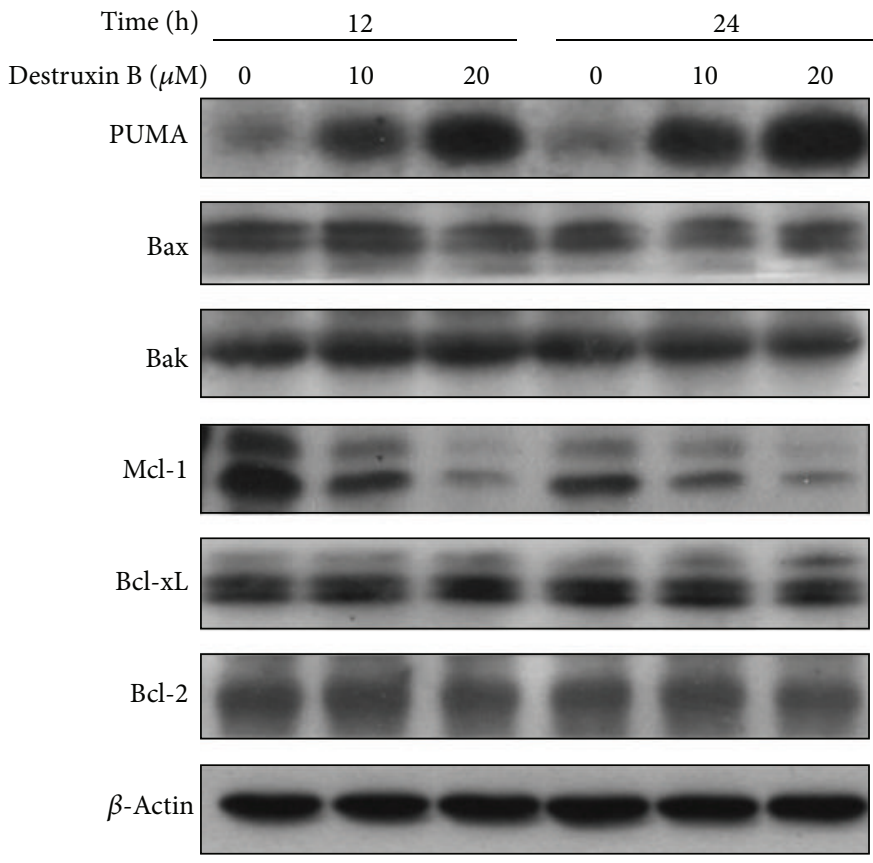

(a)
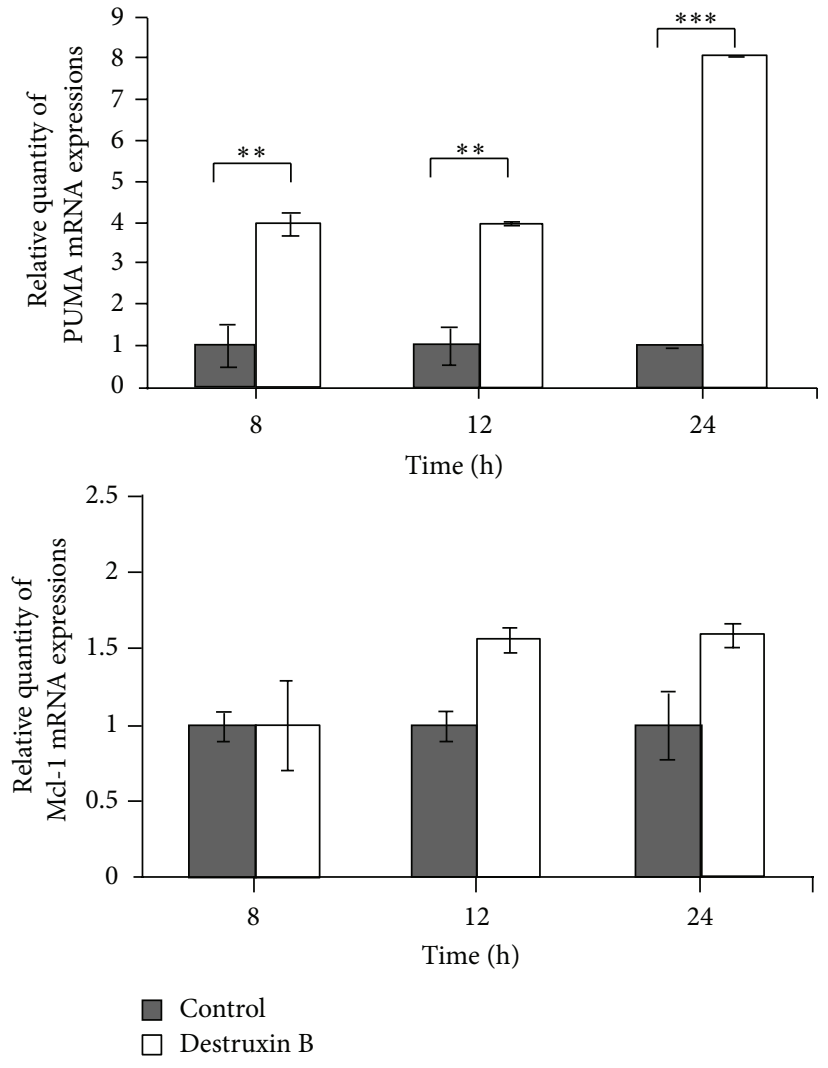

(b)

Figure 3: Regulation of Mcl-1 and PUMA by destruxin B. (a) Regulation of Bcl-2 family proteins by destruxin. A549 cells were treated with 0,10 , or $20 \mu \mathrm{M}$ of destruxin B for 12 and $24 \mathrm{~h}$. The cells were collected, lyzed, and subjected to western blot with antibodies against PUMA, Bax, Bak, Mcl-1, Bcl-xL, Bcl-2, and $\beta$-actin, respectively. (b) Upregulation of PUMA but not Mcl-1 RNA expression by destruxin. A549 cells were treated with or without $20 \mu \mathrm{M}$ destruxin B for 8,12 , and $24 \mathrm{~h}$. The cells were then collected and the total RNA was extracted followed by real-time PCR using PUMA (upper panel) or Mcl-1 (bottom panel) primer set. The actin primer set was also applied as an internal control. The normalized relative expression folds of PUMA between untreated and treated cells were quantified $\left({ }^{*} P<0.05\right.$ versus control; ${ }^{* *} P<0.01$ versus control; ${ }^{* * *} P<0.001$ versus control).

\subsection{Bcl-2 Family Molecules Contribute to Destruxin-B-} Mediated Apoptosis. It is well documented that Bcl-2 family molecule plays a central role in mitochondria-mediated apoptosis. To examine the mitochondrial apoptotic events associated with destruxin-B-induced apoptosis, we examined the levels of proapoptotic and antiapoptotic proteins by western blot analysis in whole lysates of A549 cells treated with 0,10 , and $20 \mu \mathrm{M}$ destruxin for 24 and $48 \mathrm{~h}$. It was found that destruxin B treatment markedly increased levels of the proapoptotic protein PUMA and reduced levels of antiapoptotic proteins Mcl-1 in a concentration- and timedependent manner (Figure 3(a)). However, the levels of Bax, $\mathrm{Bak}, \mathrm{Bcl}-2$, and $\mathrm{Bcl}-\mathrm{xL}$ were not affected by destruxin. To clarify whether destruxin B regulated the expression of PUMA and Mcl-1 at a transcriptional level, the mRNA expression was analyzed by qPCR. The results showed that addition of destruxin $B$ drastically increased the level of PUMA mRNA, (Figure 3(b), upper panel), strongly indicating that increasing PUMA expression by destruxin B might attribute to transcriptional regulation. In contrast, the mRNA level of Mcl-1 was not affected by destruxin B (Figure 3(b), bottom panel), suggesting that destruxin-mediated Mcl-1 protein level reduction might be regulated by a posttranslational manner.

3.4. Mitochondrial Translocation of Bax Is Important for the Destruxin-Induced Apoptosis. It has been reported that during mitochondria-mediated apoptosis, Bax can translocate from cytosol to mitochondrial membrane and form pores to disrupt the permeability of the mitochondrial membrane. We then investigated whether Bax contributed to destruxin-Bmediated mitochondrial apoptosis. Data from immunofluorescence staining showed that Bax exhibited a diffuse distribution throughout the untreated A549 cells (Figure 4(a)). However, administration of destruxin $B$ resulted in a subcellular redistribution of Bax, the immunoreactivity of Bax formed a punctate pattern, and the yellow color in the overlay of Figure 4(a) pointed out the colocalization of Bax with mitochondrial, indicating the translocation of Bax from cytosol to mitochondria upon destruxin treatment. To characterize the role of Bax in destruxin-B-induced apoptosis, we compared the effects of destruxin $\mathrm{B}$ on cell viability between 


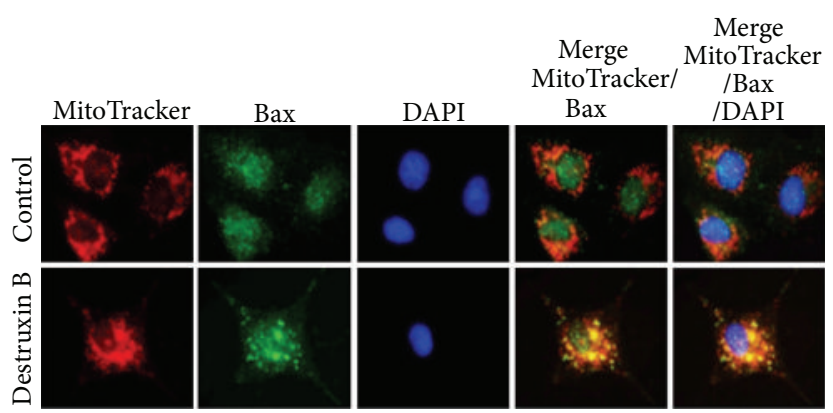

(a)

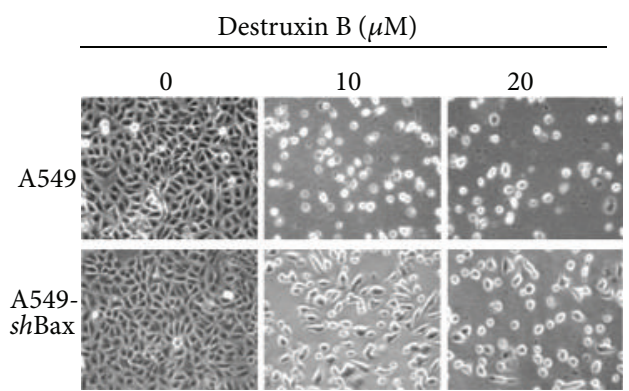

(b)
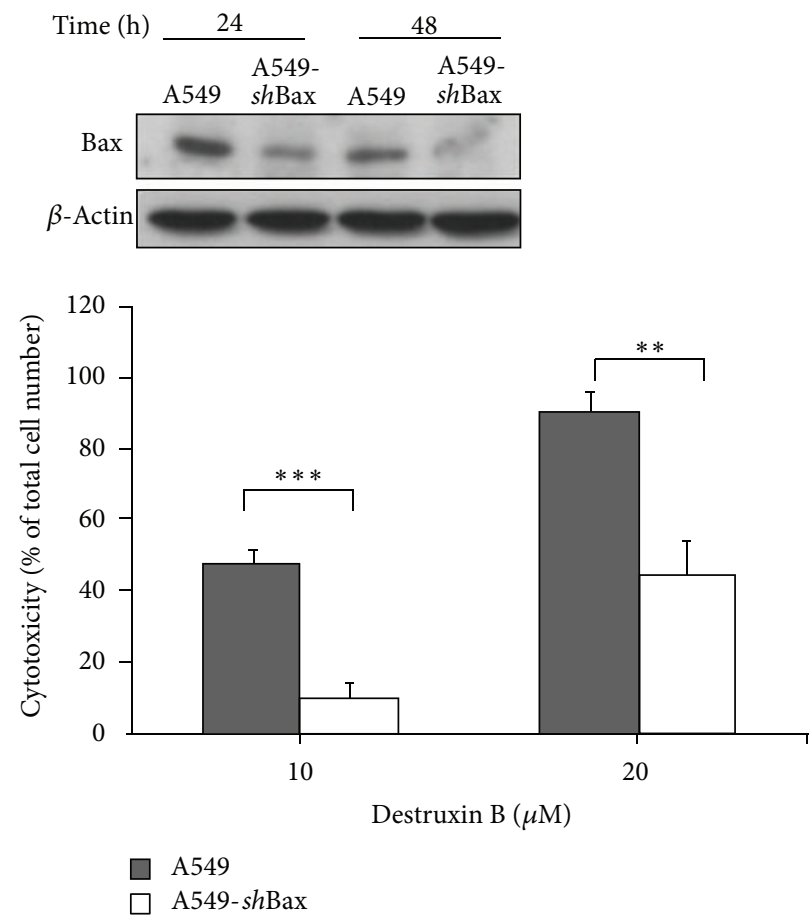

(c)

FIGURE 4: Bax plays a crucial role in destruxin-B-induced apoptosis. (a) A549 cells were untreated or treated with $20 \mu \mathrm{M}$ destruxin B for $24 \mathrm{~h}$, and the cells were then fixed with $4 \%$ paraformaldehyde and subjected to indirect immunofluorescence by using anti-Bax antibody (green color); DAPI (blue color) was used as a nuclear staining, and MitoTracker (red color) was used as a mitochondrial tracker. (b, c) The A549 and A549-shBax cells were treated with 0,10 , and $20 \mu \mathrm{M}$ destruxin B for $72 \mathrm{~h}$, (b) the cell morphology was observed under phase-contrast microscopy, and (c) the cytotoxicity of destruxin B was determined. ${ }^{* *} P<0.01$ versus control; ${ }^{* * *} P<0.001$ versus control.

parental A549 and A549-shBax cells (which were stably transfected with two Bax-specific shRNAs). As indicated in Figure 4(b), A549-shBax cells showed more resistance to the destruxin-B-induced cytotoxicity than parental A549 cells (Figures 4(b) and 4(c)). Approximately $89.9 \%$ and $43.9 \%$ apoptotic cells were detected in A549 and A549-shBax cells in response to $20 \mu \mathrm{M}$ destruxin $\mathrm{B}$ treatment, respectively. These results clearly demonstrate that $\mathrm{Bax}$ is a major proapoptotic molecule in regulation of apoptosis primed by destruxin B in A549 cells.

3.5. Destruxin B Triggers Mitochondria-Mediated Apoptosis in H1299 Cells. We expanded our study to another human lung cancer cell line, H1299. Destruxin B also inhibited cell proliferation of $\mathrm{H} 1299$ cells, with $\mathrm{IC}_{50}$ value at $4.1 \mu \mathrm{M}$ (Figure 5(a)). Consistently, the administration of destruxin B significantly stimulated the activation of caspase-2, 3 , and -9 (Figure 5(b)). Moreover, destruxin B increased the level of PUMA while decreased Mcl-1 in H1299 cells (Figure 5(c)). These results indicate that modulation of Bcl2 family molecules and activation of mitochondria-mediated caspase are required for destruxin-induced apoptosis.

\section{Discussion}

Lung cancer has been the most common malignancy in the world for several decades that carries poor prognosis. Although substantial progress has been made in developing chemotherapeutic treatments for lung cancer, drug efficacy is 


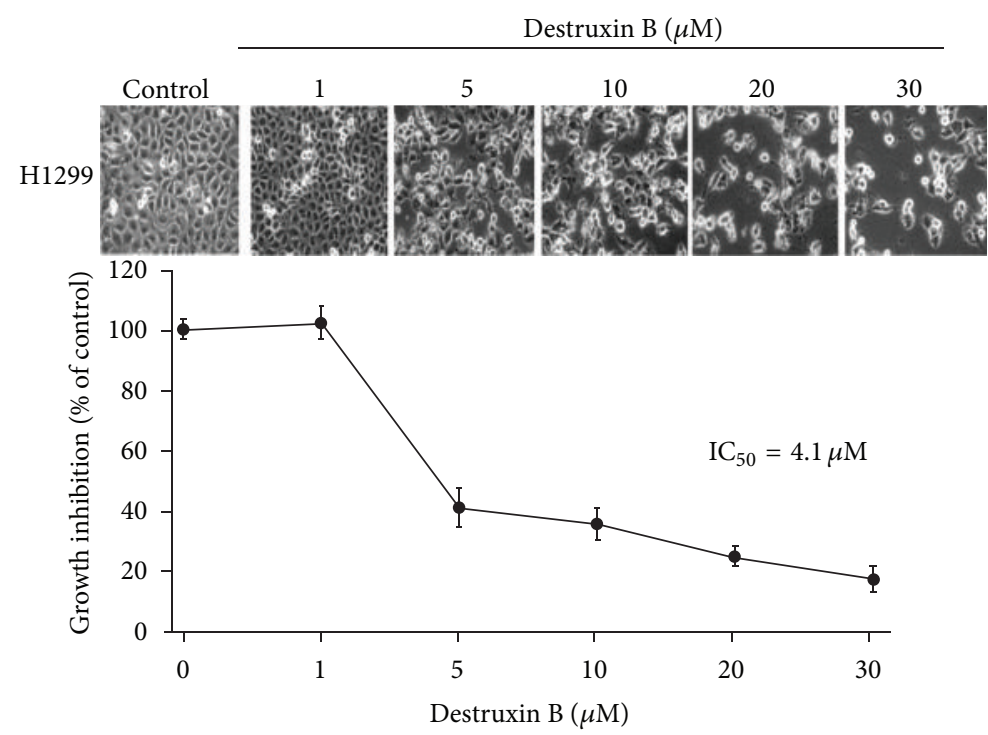

(a)

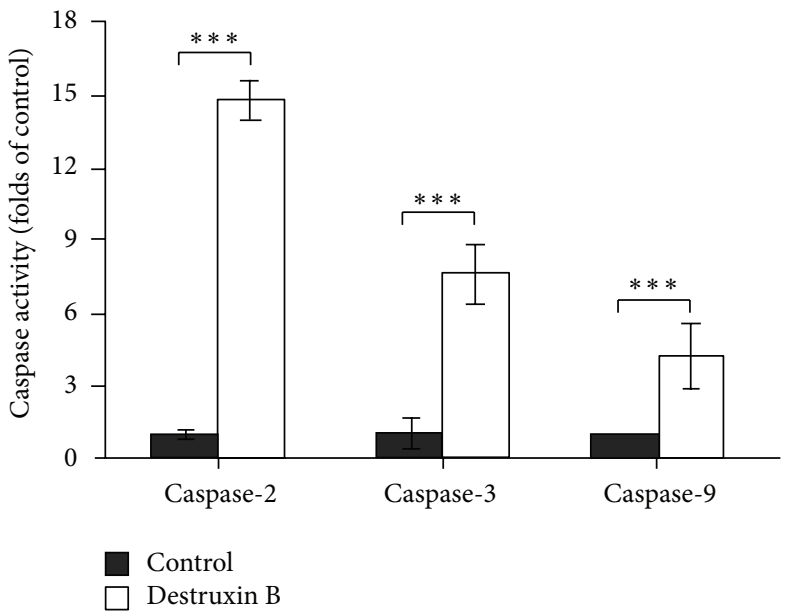

(b)

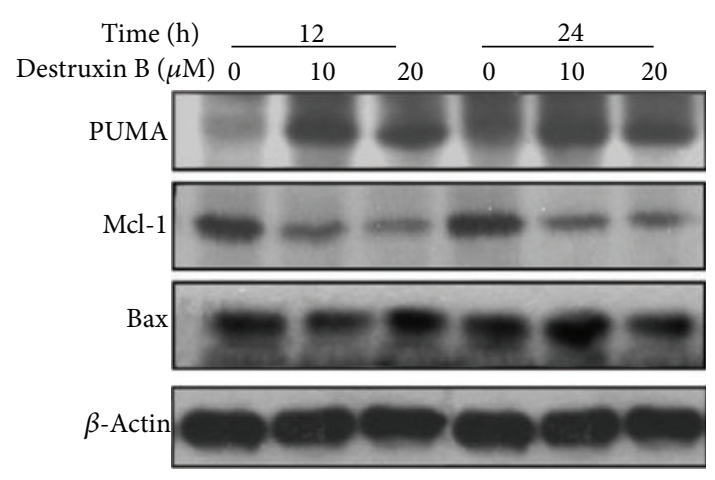

(c)

FIGURE 5: The effects of destruxin B on H1299 cells. (a) Antiproliferative effect. H1299 cells were untreated or treated with 1, 5, 10, 20, and $30 \mu \mathrm{M}$ of destruxin B for $48 \mathrm{~h}$. The cell number was measured. (b) Activation of caspases. H1299 cells were treated with $20 \mu \mathrm{M}$ destruxin $\mathrm{B}$ for $48 \mathrm{~h}$. The cells were then lyzed and incubated with the fluorescein-conjugated substrates of caspase-2, -3 , and -9 and subjected to the microplate reader to detect caspase activity. ${ }^{*} P<0.05$ versus control; ${ }^{* *} P<0.01$ versus control; ${ }^{* * *} P<0.001$ versus control. (c) H1299 cells were treated with 0,10 , or $20 \mu \mathrm{M}$ destruxin B for 12 and $24 \mathrm{~h}$. The cells were collected, lyzed, and subjected to western blot with antibodies against PUMA, Mcl-1, and $\beta$-actin, respectively.

often outweighed by undesirable side effects. Nowadays, lung cancer is still the first leading cause of death from cancer, with 1.4 million deaths annually. Thus, it is crucially important to develop better therapeutic strategies for the management of lung cancer. In the recent years, phytochemicals and microbial extracts isolated from various sources have shown significant anticancer activities [30]. It is estimated that approximately $50 \%$ of the drugs currently used in the clinic are derived from the natural products or their synthetic analogs, which still continue to provide essential sources of novel discovery leads. Cyclodepsipeptides, the secondary metabolites of fungi and plants [31], show a broad spectrum of biological activity, including immunosuppressant, antibiotic, antifungal, anti-inflammatory, and anticancer effects [31]. Some of these natural products and (semi)synthetic derivatives have already been evaluated in clinical trials [31]. Destruxin B, one of the cyclodepsipeptides, is isolated and purified from entomopathogenic fungi. Previous studies demonstrate that destruxin B has insecticidal and antiviral effects [24]. Additionally, our studies and other studies demonstrate that destruxin $\mathrm{B}$ has antitumor effect in leukemia cells [32], hepatocellular carcinoma cells [25], and colorectal cancer [33]. This study firstly reported that destruxin B inhibits cell proliferation and induces apoptotic cell death in human nonsmall cell lung cancer cell lines, A549 and H1299. Moreover, destruxin-B-triggered apoptosis 


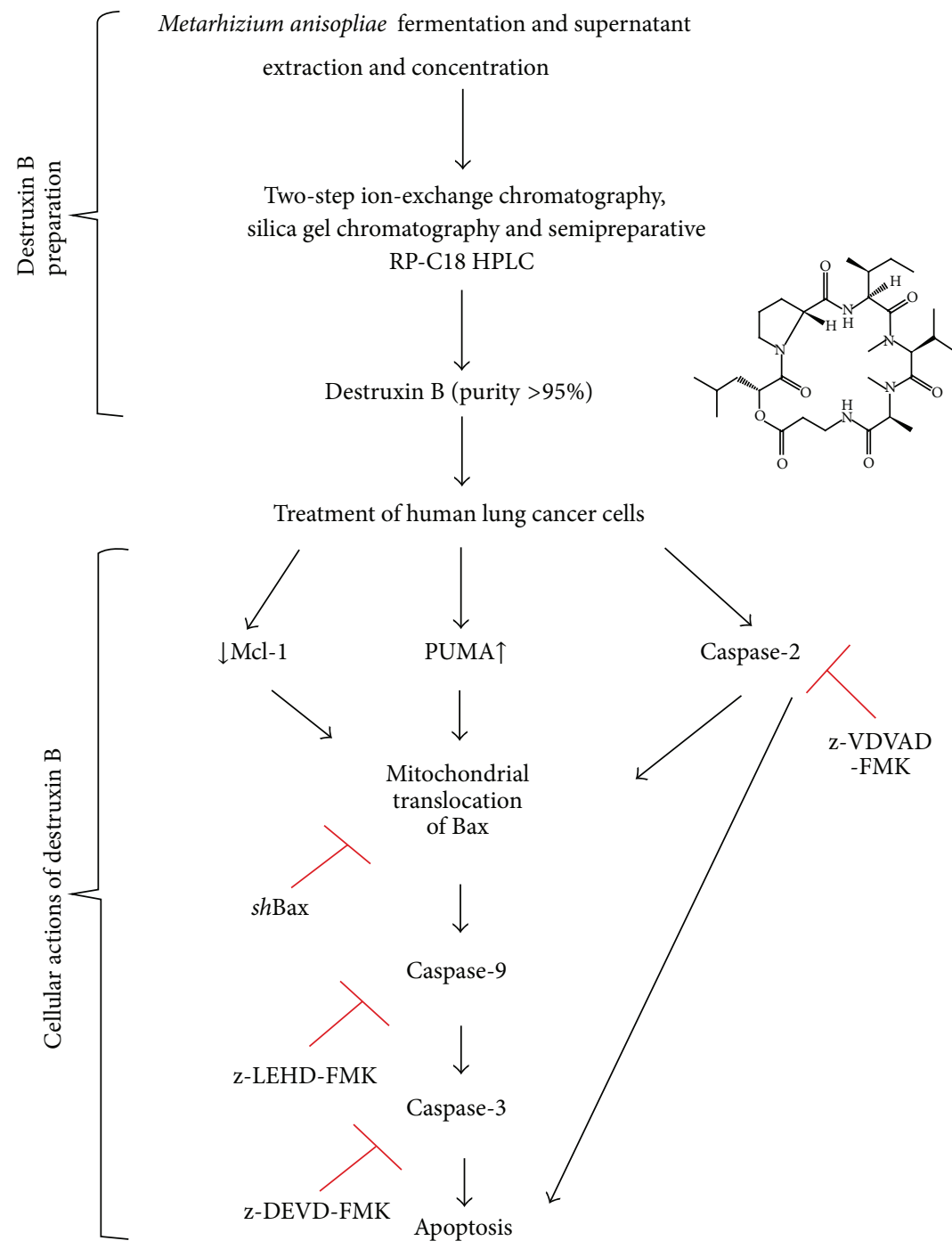

FIGURE 6: Schematic representation of destruxin B preparation and mechanistic illustration of destruxin-B-induced apoptosis. Destruxin B isolated from Metarhizium anisopliae induces apoptosis in human nonsmall cell lung cancer cells by activating a mitochondrial-dependent caspase activation signaling pathway.

is through a Bax-dependent mitochondria-mediated caspase activation pathway.

Our paper is the first report to conduct a mechanistic study to investigate how destruxin B induces apoptosis. It is well documented that apoptosis is activated mainly through two pathways: the mitochondria-dependent pathway (intrinsic pathway) and the death receptor pathway (extrinsic pathway). Bcl-2 family proteins play a key role in the regulation of mitochondria-dependent apoptosis [34]. The relative equilibrium of various anti- and proapoptotic Bcl-2 family members are a critical determinant of cell apoptosis. Bax is mainly localized in the cytosol of healthy cells as a soluble monomeric protein in an apoptotically inactive status [35]. In response to apoptotic cues, Bax undergoes conformational changes and then translocates to the mitochondrial outer membrane and forms large oligomeric complexes, thereby triggering mitochondrial dysfunction and caspase activation to bring about apoptosis. Mcl-1 can directly bind with
Bax and prevent apoptotic activation of Bax [36]. In this study, we found that treatment with destruxin $B$ resulted in decrease of Mcl-1, increase of PUMA, and induction of Bax mitochondrial translocation. Moreover, silencing Bax expression by shRNA effectively attenuated destruxin-Btriggered apoptosis. This suggests that Bax plays a more crucial role in conducting destruxin-B-mediated cytotoxicity. It is known that PUMA is a general sensor of apoptotic stimuli and a promising drug target for cancer therapy [37]. Biochemical studies indicate that PUMA induces apoptosis by activating the proapoptotic protein Bax through its interaction with antiapoptotic Bcl-2 family members, including $\mathrm{Bcl}-2, \mathrm{Bcl}-\mathrm{xL}, \mathrm{Mcl}-1, \mathrm{Bcl}-\mathrm{w}$, and A1 [38]. The interactions of PUMA with antiapoptotic proteins cause displacement of Bax, resulting in activation of the proapoptotic activity of Bax. PUMA overexpression leads to a conformational change, multimerization, and mitochondrial translocation of Bax, which induces apoptotic cell death [37]. Based on 
these data, we propose that one of the mechanisms by which destruxin-B-induced apoptosis was by overexpression of PUMA and decrease of Mcl-1 resulting in disruption of Mcl-1/Bax complexes, triggering Bax oligomerization and translocation to mitochondria in human nonsmall cell lung cancer cells.

The apoptosis process is mediated by sequential activation of caspases. Caspases, a family of cysteine proteases, is one of the biomarkers of apoptosis [39]. Once activated, caspases can cleave hundreds of cellular proteins resulting in the hallmark morphological characteristics of apoptosis. Accumulating evidence indicates that activation of caspase cascade plays a key role in the induction of apoptosis for many cancer therapeutic drugs in many types of cancer cells [39]. In particular, activation of caspase-3 plays a central role in the execution of apoptosis. Activation of caspase-3 requires the activation of initiator caspases, such as caspase8 or caspase- 9 , in response to proapoptotic signals. It is well known that caspase- 9 is the major initiator caspase of mitochondria-mediated apoptosis while caspase- 8 is the key initiator caspase of death receptor-triggered apoptosis. Our current study clearly demonstrated that treatment of A549 cells with destruxin B enhanced the activation of caspase- 9 and -3 . Reduction of caspase cascade with specific inhibitors effectively blocked destruxin-B-induced apoptosis. These results indicated that destruxin-B-induced apoptosis was through a mitochondria-dependent caspase-9/-3-activated response. Interestingly, this study also showed that caspase2 was activated in A549 and H1299 cells upon destruxin B administration. Caspase- 2 is considered as both initiator and executor caspase in apoptosis [40]. Caspase- 2 has been shown to act upstream of the mitochondria and is involved in Bid cleavage and Bax translocation, which results in cytochrome $c$ release during genotoxic stress [41]. Therefore, we proposed that the activation of caspase- 2 by destruxin $B$ might be one of the important regulators in inducing Bax translocation and subsequent mitochondrial dysfunction, finally leading to apoptosis.

In conclusion, the present study supports that destruxin B isolated and purified from Metarhizium anisopliae F061 var. Anisopliae induces the intrinsic apoptotic pathway in human nonsmall cell lung cancer A549 cells, and a signaling cascade is proposed in Figure 6. Destruxin-B-mediated upregulation of PUMA and downregulation of Mcl-1 may facilitate the mitochondrial translocation of Bax, subsequently inducing caspase-2, -9, and -3 activation and apoptosis. Importantly, inhibition of Bax expression by shRNA significantly diminishes destruxin-B-induced cytotoxicity, suggesting that Baxdependent mitochondrial pathway contributes to destruxin B activity in A549 cells. Because some of these results have been confirmed in H1299 cells, we hypothesize that destruxin B may activate some common signaling events in different human nonsmall cell lung cancer cell types.

\section{Conflict of Interests}

The authors declare that there is no potential conflict of interests.

\section{Acknowledgments}

This work was supported by the National Science Council of Taiwan (NSC 100-2113-M-324-001-MY3), the Taichung Veteran General Hospital Taichung, and National Chi Nan University (TCVGH-NCNU1017901).

\section{References}

[1] A. Jemal, R. Siegel, E. Ward, Y. Hao, J. Xu, and M. J. Thun, "Cancer statistics, 2009," CA: Cancer Journal for Clinicians, vol. 59, no. 4, pp. 225-249, 2009.

[2] A. Jemal, F. Bray, M. M. Center, J. Ferlay, E. Ward, and D. Forman, "Global cancer statistics," CA: Cancer Journal for Clinicians, vol. 61, no. 2, pp. 69-90, 2011.

[3] M. R. Stratton, P. J. Campbell, and P. A. Futreal, "The cancer genome," Nature, vol. 458, no. 7239, pp. 719-724, 2009.

[4] M. G. Kris, R. B. Natale, R. S. Herbst et al., "Efficacy of gefitinib, an inhibitor of the epidermal growth factor receptor tyrosine kinase, in symptomatic patients with non-small cell lung cancer: a randomized trial," Journal of the American Medical Association, vol. 290, no. 16, pp. 2149-2158, 2003.

[5] S. Elmore, "Apoptosis: a review of programmed cell death," Toxicologic Pathology, vol. 35, no. 4, pp. 495-516, 2007.

[6] T. S. Mok, Y.-L. Wu, S. Thongprasert et al., "Gefitinib or carboplatin-paclitaxel in pulmonary adenocarcinoma," The New England Journal of Medicine, vol. 361, no. 10, pp. 947-957, 2009.

[7] T. G. Cotter, "Apoptosis and cancer: the genesis of a research field," Nature Reviews Cancer, vol. 9, no. 7, pp. 501-507, 2009.

[8] C. Wang and R. J. Youle, "The role of mitochondria in apoptosis," Annual Review of Genetics, vol. 43, pp. 95-118, 2009.

[9] T. J. Sayers, "Targeting the extrinsic apoptosis signaling pathway for cancer therapy," Cancer Immunology, Immunotherapy, vol. 60, no. 8, pp. 1173-1180, 2011.

[10] P. Katiyar, M. Yadav, and A. Tiwari, "Role of caspase in programmed cell death in multicellular organisms," International Journal of Biomedical Research, vol. 2, no. 7, pp. 422-431, 2011.

[11] I. Budihardjo, H. Oliver, M. Lutter, X. Luo, and X. Wang, "Biochemical pathways of caspase activation during apoptosis," Annual Review of Cell and Developmental Biology, vol. 15, no. 1, pp. 269-290, 1999.

[12] G. Kroemer, "The proto-oncogene Bcl-2 and its role in regulating apoptosis," Nature Medicine, vol. 3, no. 6, pp. 614-620, 1997.

[13] Y. Rong and C. W. Distelhorst, "Bcl-2 protein family members: versatile regulators of calcium signaling in cell survival and apoptosis," Annual Review of Physiology, vol. 70, pp. 73-91, 2008.

[14] R. J. Youle and A. Strasser, "The BCL-2 protein family: opposing activities that mediate cell death," Nature Reviews Molecular Cell Biology, vol. 9, no. 1, pp. 47-59, 2008.

[15] Y.-L. P. Ow, D. R. Green, Z. Hao, and T. W. Mak, "Cytochrome c: functions beyond respiration," Nature Reviews Molecular Cell Biology, vol. 9, no. 7, pp. 532-542, 2008.

[16] J. W.-H. Li and J. C. Vederas, "Drug discovery and natural products: end of an era or an endless frontier?" Science, vol. 325, no. 5937, pp. 161-165, 2009.

[17] G. M. Cragg, P. G. Grothaus, and D. J. Newman, "Impact of natural products on developing new anti-cancer agents," Chemical Reviews, vol. 109, no. 7, pp. 3012-3043, 2009.

[18] J. Kopecký, V. Matha, and A. Jegorov, “The inhibitory effect of destruxin a on the replication of arboviruses in Aedes albopictus 
C6/36 cell line," Comparative Biochemistry and Physiology C, vol. 103, no. 1, pp. 23-25, 1992.

[19] K. Adachi, H. Nanba, and H. Kuroda, "Potentiation of hostmediated antitumor activity in mice by $\beta$-glucan obtained from Grifola frondosa (maitake)," Chemical and Pharmaceutical Bulletin, vol. 35, no. 1, pp. 262-270, 1987.

[20] T. Ohmori, K. Tamura, S. Tsuru, and K. Nomoto, "Antitumor activity of protein-bound polysaccharide from Cordyceps ophioglossoides in mice," Japanese Journal of Cancer Research, vol. 77, no. 12, pp. 1256-1263, 1986.

[21] R. E. Moore, "Cyclic peptides and depsipeptides from cyanobacteria: a review," Journal of Industrial Microbiology, vol. 16, no. 2, pp. 134-143, 1996.

[22] K. L. Rinehart, "Antitumor compounds from tunicates," Medicinal Research Reviews, vol. 20, no. 1, pp. 1-27, 2000.

[23] G. A. Bradfisch and S. L. Harmer, " $\omega$-conotoxin GVIA and nifedipine inhibit the depolarizing action of the fungal metabolite, destruxin B on muscle from the tobacco budworm (heliothis virescens)," Toxicon, vol. 28, no. 11, pp. 1249-1254, 1990.

[24] H.-C. Chen, C.-K. Chou, C.-M. Sun, and S. F. Yeh, "Suppressive effects of destruxin $\mathrm{B}$ on hepatitis $\mathrm{B}$ virus surface antigen gene expression in human hepatoma cells," Antiviral Research, vol. 34, no. 3, pp. 137-144, 1997.

[25] F. Odier, A. Vey, and J. P. Bureau, "In vitro effect of fungal cyclodepsipeptides on leukemic cells: study of destruxins A, B and E," Biology of the Cell, vol. 74, no. 3, pp. 267-271, 1992.

[26] J.-W. Chen, B.-L. Liu, and Y.-M. Tzeng, "Purification and quantification of destruxins A and B from Metarhizium anisopliae," Journal of Chromatography A, vol. 830, no. 1, pp. 115-125, 1999.

[27] B. L. Liu, T. M. Rou, Y. K. Rao, and Y. M. Tzeng, "Effect of $\mathrm{pH}$ and aeration rate on the production of destruxins $\mathrm{A}$ and $\mathrm{B}$ from Metarhizium anisopliae," International Journal of Applied Science and Engineering, vol. 5, no. 1, pp. 17-26, 2007.

[28] Y. P. Lee, C. W. Wang, W. C. Liao et al., "In vitro and in vivo anticancer effects of destruxin B on human colorectal cancer," Anticancer Research, vol. 32, no. 7, pp. 2735-2745, 2012.

[29] G.-C. Chang, C.-T. R. Yu, C.-H. Tsai et al., "An epidermal growth factor inhibitor, Gefitinib, induces apoptosis through a p53-dependent upregulation of pro-apoptotic molecules and downregulation of anti-apoptotic molecules in human lung adenocarcinoma A549 cells," European Journal of Pharmacology, vol. 600, no. 1-3, pp. 37-44, 2008.

[30] B. S. Patil, G. K. Jayaprakasha, K. N. Chidambara Murthy, and A. Vikram, "Bioactive compounds: historical perspectives, opportunities and challenges," Journal of Agricultural and Food Chemistry, vol. 57, no. 18, pp. 8142-8160, 2009.

[31] R. Lemmens-Gruber, M. R. Kamyar, and R. Dornetshuber, "Cyclodepsipeptides-potential drugs and lead compounds in the drug development process," Current Medicinal Chemistry, vol. 16, no. 9, pp. 1122-1137, 2009.

[32] E. Morel, M. Pais, M. Turpin, and M. Guyot, "Cytotoxicity of cyclodepsipeptides on murine lymphocytes and on L 1210 leukemia cells," Biomedicine and Pharmacotherapy, vol. 37, no. 4, pp. 184-185, 1983.

[33] C.-T. Yeh, Y. K. Rao, M. Ye et al., "Preclinical evaluation of destruxin $\mathrm{B}$ as a novel Wnt signaling target suppressing proliferation and metastasis of colorectal cancer using noninvasive bioluminescence imaging," Toxicology and Applied Pharmacology, vol. 261, no. 1, pp. 31-41, 2012.

[34] R. M. Kluck, E. Bossy-Wetzel, D. R. Green, and D. D. Newmeyer, "The release of cytochrome $\mathrm{c}$ from mitochondria: a primary site for Bcl- 2 regulation of apoptosis," Science, vol. 275, no. 5303, pp. 1132-1136, 1997.

[35] B. Leber, J. Lin, and D. W. Andrews, "Embedded together: the life and death consequences of interaction of the Bcl-2 family with membranes," Apoptosis, vol. 12, no. 5, pp. 897-911, 2007.

[36] A. Cuconati, C. Mukherjee, D. Perez, and E. White, "DNA damage response and MCL-1 destruction initiate apoptosis in adenovirus-infected cells," Genes and Development, vol. 17, no. 23, pp. 2922-2932, 2003.

[37] J. Yu and L. Zhang, "PUMA, a potent killer with or without p53," Oncogene, vol. 27, no. 1, pp. S71-S83, 2008.

[38] L. Chen, S. N. Willis, A. Wei et al., "Differential targeting of prosurvival $\mathrm{Bcl}-2$ proteins by their $\mathrm{BH} 3$-only ligands allows complementary apoptotic function," Molecular Cell, vol. 17, no. 3, pp. 393-403, 2005.

[39] M. S. Ola, M. Nawaz, and H. Ahsan, "Role of Bcl-2 family proteins and caspases in the regulation of apoptosis," Molecular and Cellular Biochemistry, vol. 351, no. 1-2, pp. 41-58, 2011.

[40] T.-J. Fan, L.-H. Han, R.-S. Cong, and J. Liang, "Caspase family proteases and apoptosis," Acta Biochimica et Biophysica Sinica, vol. 37, no. 11, pp. 719-727, 2005.

[41] S. Kumar, "Caspase 2 in apoptosis, the DNA damage response and tumour suppression: enigma no more?" Nature Reviews Cancer, vol. 9, no. 12, pp. 897-903, 2009. 


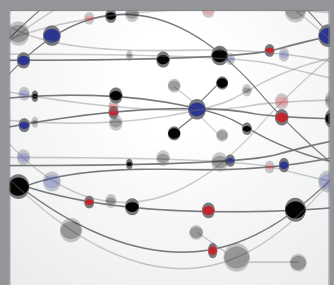

The Scientific World Journal
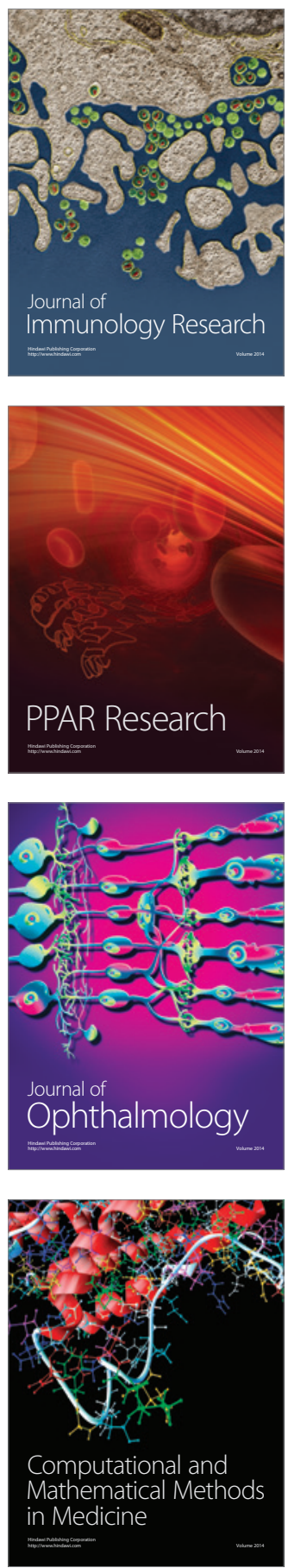

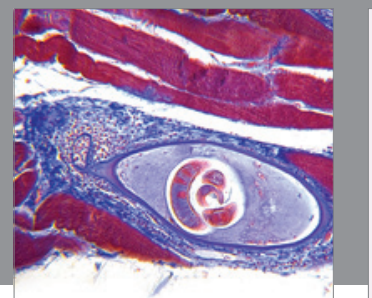

Gastroenterology

Research and Practice
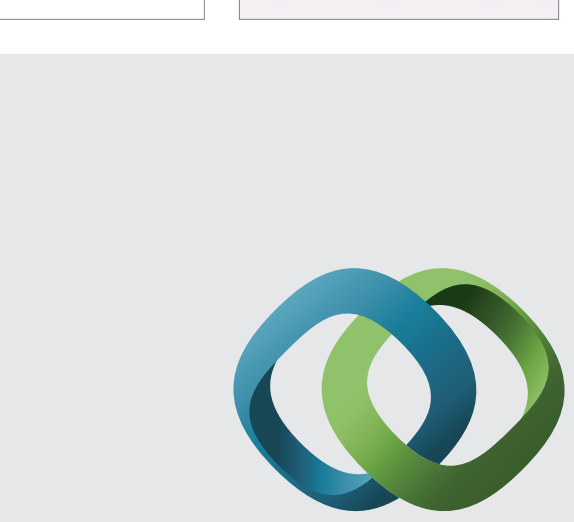

\section{Hindawi}

Submit your manuscripts at

http://www.hindawi.com
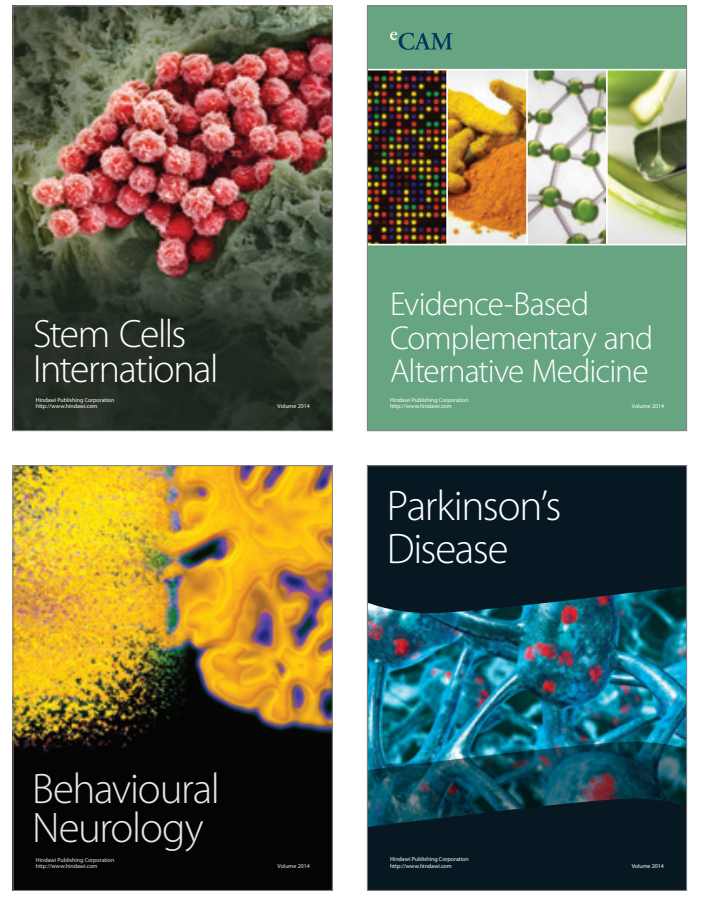
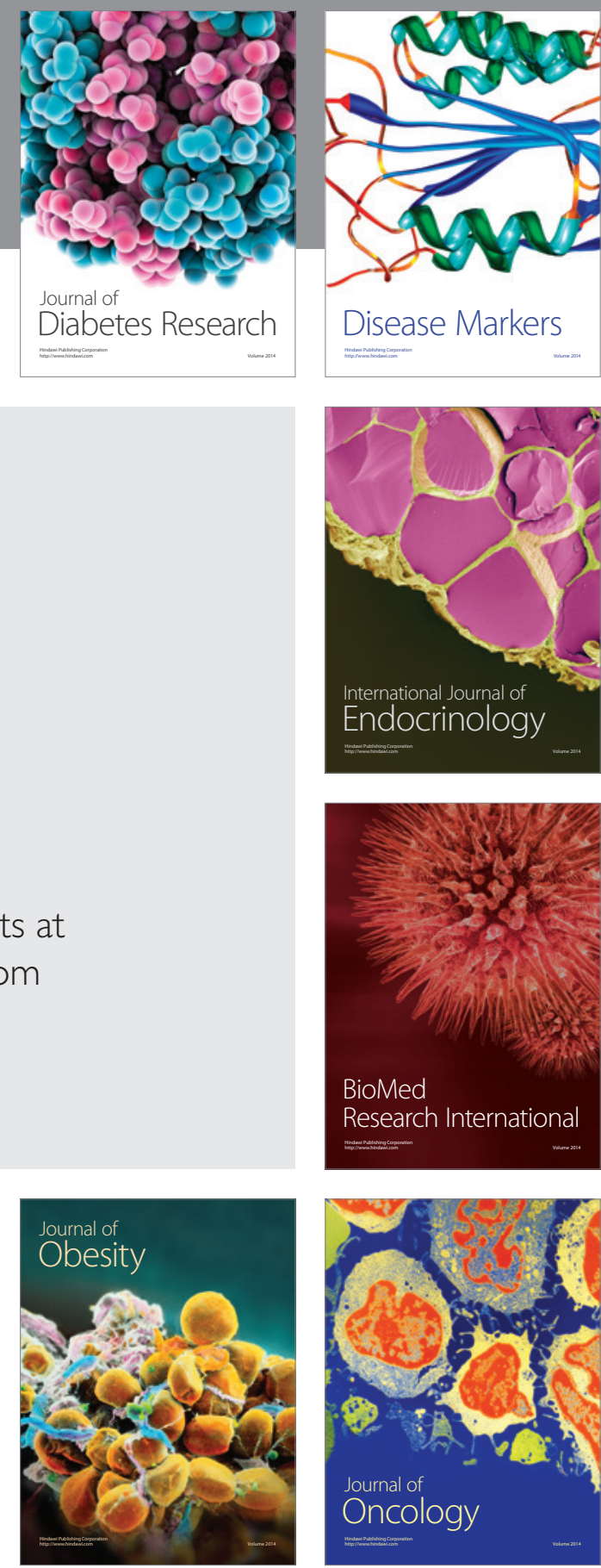

Disease Markers
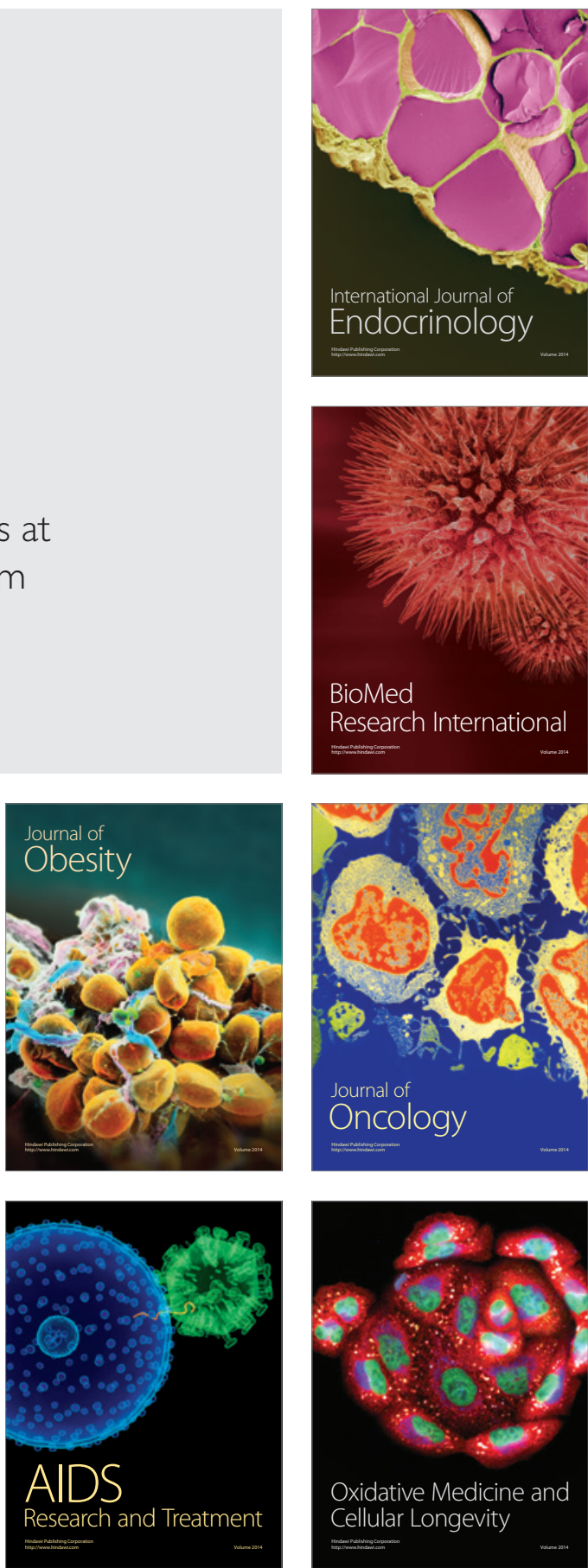